OBSERVATION OF A FOUR-PION VECTOR-MESON STATE

OF MASS $\cong 1.5 \mathrm{GeV}$ PRODUCED BY

LINEARLY POLARIZED $9.3 \mathrm{GeV}$ PHOTONS*

H. H. Bingham, W. B. Fretter, W. J. Podolsky, **

M. S. Rabin, *** A. H. Rosenfeld, G. Smadja, $t$ and G. P. Yost

University of California and Lawrence Berkeley Laboratory Berkeley, California 94720

and

J. Ballam, G. B. Chadwick, Y. Eiscnberg, $\dagger \dagger$ E. Kogan, t†

K. C. Moffeit, P. Seyboth, $t \dagger \dagger$ I. O. Skillicorn, $\neq$

H. Spitzer, $\neq \neq$ and G. Wolf $\neq \neq \neq$

Stanford Linear Accelerator Center

Stanford University, Stanford, California 94305

\title{
ABSTRACT
}

From a study of $\gamma \mathrm{p}-2 \pi^{+} 2 \pi^{-} \mathrm{p}$ using $9.3 \mathrm{GeV}$ linearly polarized photons, evidence is found for a $\mathrm{J}^{\mathrm{P}}=1^{-}, \mathrm{I}^{\mathrm{G}}=1^{+}$four pion enhancement of mass $\sim 1.5 \mathrm{GeV}$ with a production cross section of $1.6 \pm 0.4 \mu \mathrm{b}$.

(Submitted to Physics Letters.)

*Work supported in part by the U. S. Atomic Energy Commission and National Science Foundation.

**Present address: University of Washington, Seattle, Wash.

***Present address: University of Massachusetts, Amherst, Mass.

$\dagger$ Fellow of the Miller Institute for Basic Research in Science, on leave from

DPHPE, CEN, Saclay, France.

††On leave from the Weizmann Institute, Rehovoth, Israel.

t††Present address: Max-Planck-Institut für Physik und Astrophysik, München.

$\ddagger$ Present address: University of Glasgow, Physics Dept., Glasgow.

$\neq \ddagger$ Present address: University of Hamburg, Hamburg, Germany。

$\neq \neq \neq$ Present address: DESY, Hamburg, Germany. 
Previous indications of possible vector meson states, other than $\rho, \omega$, and $\Phi$, have been wide enhancements near $1.6 \mathrm{GeV}$ in $\gamma p \rightarrow 2 \pi^{+} 2 \pi^{-} p$ and in $\mathrm{e}^{+} \mathrm{e}^{-} \rightarrow 4 \pi$ (+ neutrals). ${ }^{1,2}$ We confirm the existence of a $4 \pi$ enhancement at $\sim 1.5 \mathrm{GeV}$ in the reaction $\gamma \mathrm{p} \rightarrow 2 \pi^{+} 2 \pi^{-} \mathrm{p}$ and demonstrate, using the linear polarization of the beam, that it is in a $J^{\mathrm{P}}=1^{-}, I^{\mathrm{G}}=1^{+}$state. Preliminary results have been reported elsewhere. ${ }^{3}$

The data presented here originate from a systematic study ${ }^{4}$ of $\gamma p$ reactions at $2.8,4.7$, and $9.3 \mathrm{GeV}$, using the LBL-SLAC $82 " \mathrm{HBC}$ exposed to the SLAC monochromatic backscattered laser beam. ${ }^{5,6}$ The $4 \pi$ enhancement is observed most clearly at $9.3 \mathrm{GeV}$, and therefore we present results based on this exposure. At $9.3 \mathrm{GeV}$ the beam momentum spread is $\Delta p / p \approx \pm 3 \%$, and the degree of linear polarization, $\mathrm{P}_{\gamma}$, is $77 \%$. The event analysis is described in Ref. 6 . From a total of 1.2 million pictures we obtained 1041 events of the reaction

$$
\gamma p \rightarrow 2 \pi^{+} 2 \pi^{-} \mathrm{p}
$$

with $8.0<\mathrm{E}_{\gamma}<10.3 \mathrm{GeV}$ and a kinematic confidence level greater than $1 \%$. The channel cross section is $4.1 \pm 0.2 \mu \mathrm{b} .^{6}$

Mass Distributions

The $4 \pi$ mass distribution for reaction (1) is shown in Fig. 1a. From the t-distribution (not shown) we find that $4 \pi$ production is peripheral with a $t$ dependence $\sim \mathrm{e}^{6 \mathrm{t}}$ for $\mathrm{M}_{4 \pi}<2.4 \mathrm{GeV}$ ( $\mathrm{t}$ is the momentum transfer squared to the proton). Phase space weighted by this t-distribution peaks at a $4 \pi$ mass $\sim 2.5 \mathrm{GeV}$. The reflection from $\Delta^{++}$production, which is strong in reaction (1) (see Fig. 1b), also peaks at high $4 \pi$ mass. In contrast, the $4 \pi$ mass spectrum shown in Fig. 1a is enhanced at $\sim 1.5 \mathrm{GeV}$.

Figure $1 \mathrm{~b}$ shows the $\pi^{+} \mathrm{p}$ mass distribution; events having $\mathrm{M}_{4 \pi}<1.7 \mathrm{GeV}$ are shaded. There is no $\Delta^{++}$peak present for low $4 \pi$ masses. By removing 
$\Delta^{++}$events from reaction (1) we find an upper limit of $2.1 \pm 0.2 \mu \mathrm{b}$ for the cross section of the $4 \pi$ enhancement (shaded events of Fig. 1a).

A strong $\rho^{0}$ signal is present in the $\pi^{+} \pi^{-}$mass distribution (Fig. 1d). The shaded portion of Fig. Id demonstrates that $\rho{ }^{0}$ production also occurs in the low mass $4 \pi$ enhancement. Figure 1c shows the $\pi^{+} \pi^{-}$mass distribution, for $\mathrm{M}_{4 \pi}<1.7 \mathrm{GeV}$, when the opposite $\pi^{+} \pi^{-}$mass combination is in the rho region. There is no evidence for a substantial contribution of $\rho^{0} \rho^{0}$. This is confirmed by a maximum-likelihood fit, with incoherent $\rho^{\circ} \rho^{\circ}$ (dashed curve on Fig. 1c), $\rho^{\circ} \pi^{+} \pi^{-}$, and phase space, to the mass distribution of events with $\mathrm{M}_{4 \pi}<1.7 \mathrm{GeV}$; the fit required no $\rho^{\circ} \rho^{\circ}, \sim 80 \% \rho^{\circ} \pi^{+} \pi^{-}$, and $\sim 20 \%$ phase space. The solid curve on Fig. 1c shows the result of this fit.

\section{Angular Distribution}

We have shown ${ }^{3}$ that in the $4 \pi$ rest frame, the vector $\vec{Q}=\vec{P}_{\pi_{1}^{+}}+\vec{P}_{\pi_{2}^{+}}$may be a good analyzer for a $\mathrm{J}^{\mathrm{P}}=1^{-}, \mathrm{I}^{\mathrm{G}}=1^{+}, 4 \pi$ state. Following our procedures introduced for vector-meson analysis, ${ }^{4}$ we give in Fig. $2 \mathrm{a}$, for $\mathrm{M}_{4 \pi}<1.7 \mathrm{GeV}$, the distribution of the angles $\theta, \psi$ calculated $^{7}$ in the helicity frame using the analyzer $\vec{Q}$. Strong $\sin ^{2} \theta$ and $\cos ^{2} \psi$ components, characteristic of the decay of an s-channel helicity-conserving vector meson produced via natural-parity exchange, are evident in the angular distribution. A similar plot in the Gottfried-Jackson frame (not shown) shows weaker $\sin ^{2} \theta$ and $\cos ^{2} \psi$ terms indicating that, like the $\rho^{\circ}$ in $\gamma \mathrm{p} \rightarrow \mathrm{p} \rho^{\circ}$, the $4 \pi$ system is more strongly aligned in the helicity frame.

The amount of $\sin ^{2} \theta \cos ^{2} \psi$ component in the $4 \pi$ angular distribution, and consequently the number of s-channel helicity-conserving $\mathrm{J}^{\mathrm{P}}=1^{-} 4 \pi$ events, can be determined ${ }^{4}$ from

$$
\Pi=\frac{1}{\mathrm{P}_{\gamma}} \sqrt{\frac{40 \pi}{3}} \Sigma \operatorname{Re} \mathrm{Y}_{2}^{2}(\theta, \psi)
$$


if $\vec{Q}$ is a perfect analyzer. The quantity $\Pi$ is shown as a function of $4 \pi$ mass in Fig. 2b; II peaks near $1.5 \mathrm{GeV}$ and has a FWHM of $\sim 0.5 \mathrm{GeV}$. We note that only the $\mathrm{Y}_{2}^{0}$, and $\mathrm{Y}_{2}^{2}$ moments are significant for $\mathrm{M}_{4 \pi}<1.8 \mathrm{GeV}$. Thus our data are consistent with a $\mathrm{J}^{\mathrm{P}}=1^{-} 4 \pi$ enhancement which we refer to as the $\rho^{\prime}$. The cross section corresponding to $\Pi$, which is a lower limit for the $\rho^{\prime}$ cross section, is $0.8 \pm 0.2 \mu \mathrm{b}$.

Isospin

We have shown that the $\rho^{\prime}$ decays predominantly into $\rho^{0} \pi^{+} \pi^{-}$. The $\pi^{+} \pi^{-}$ pair could have isospin 0,1 , or 2 . We can rule out the dominance of $I=2$ for the dipion state since it requires $R=\left(\rho^{\prime} \rightarrow \rho \pi \pi \rightarrow \pi^{+} \pi^{-} \pi^{0} \pi^{0}\right) /\left(\rho^{\prime} \rightarrow \rho^{0} \pi^{+} \pi^{-}\right) \geq 4$, which is incompatible with the observed number of events in the $\rho^{\prime}$ region in the channel $\gamma \mathrm{p} \rightarrow \mathrm{p} \pi^{+} \pi^{-} \mathrm{MM}$ (not shown). Isospin 1 for the dipion pair implies a $\rho^{\circ} \rho^{\circ}$ decay, which is inconsistent with the dipion mass distribution (Fig. 1c). Thus the $\pi^{+} \pi^{-}$pair is likely to be in a predominantly isospin zero state, giving a branching ratio $R \simeq 0.5$, which is consistent with our data. We conclude that the $\rho^{\prime}$ isospin is $1(\mathrm{C}=-1)$; this allows us to associate the $\rho^{\prime}$ with the state observed in $\mathrm{e}^{+} \mathrm{e}^{-}$experiments.

Spin-Parity Analysis

We have made a spin-parity analysis for events with $\mathrm{M}_{4 \pi}<1.7 \mathrm{GeV}$, assuming that the $4 \pi$ system is produced conserving s-channel helicity and that it decays into $\rho^{0}+\sigma$, where $\sigma$ is an s-wave isoscalar $\pi \pi$ state. Further, we must have natural (unnatural) parity $\mathrm{t}$-channel exchanges in the production of natural (unnatural) parity $4 \pi$ states in order to reproduce the experimental $\cos ^{2} \psi$ distribution.

The angular distribution of $\vec{Q}$ has been calculated ${ }^{8}$ for the states $1^{ \pm}, 2^{ \pm}$, and $3^{-}$assuming the lowest allowed angular momentum between the $\rho^{\circ}$ and $\sigma$. 
The states $1^{+}, 2^{+}$and $3^{-}$yield $\cos ^{2} \theta$ and $\cos ^{2} \psi$ components in contrast to the experimentally observed $\sin ^{2} \theta$ and $\cos ^{2} \psi$ distributions. States with helicity other than \pm 1 could introduce $\sin ^{2} \theta$ terms but only with a reduction in the amount of the $\cos ^{2} \psi$ component. Thus we rule out the dominance of the $1^{+}, 2^{+}$ and $3^{-}$states in the low $M_{4 \pi}$ region. Both $1^{-}$and $2^{-}$give angular distributions having large $\sin ^{2} \theta$ and $\cos ^{2} \psi$ components; to determine which of these states fits the data best, a maximum-likelihood fit was made with a matrix element consisting of an incoherent sum of $1^{-}, 2^{-}$and phase space. Specifically, assuming the simplest form for the decay of a $1^{-}$state into $\rho^{\circ} \pi^{+} \pi^{-}$, we write the matrix element for production of an s-channel helicity-conserving $\rho^{\prime}$, followed by its s-wave decay into a $\rho^{\circ}$ and an s-wave isospin-zero dipion system, as

$$
\mathrm{T}_{\rho^{\prime}}=\overrightarrow{\mathscr{E}} \cdot \sum_{i=1}^{4} \overrightarrow{\mathrm{q}}_{\mathrm{i}} \frac{\sin \delta_{i}^{\mathrm{p}} \mathrm{e}^{\mathrm{i} \delta_{i}^{\mathrm{p}}}}{\mathrm{q}_{\mathrm{i}}^{3}} \cdot \frac{\sin \delta^{\mathrm{s}} \mathrm{e}^{\mathrm{i} \delta^{\mathrm{s}}}}{\mathrm{q}}
$$

where $\overrightarrow{\mathscr{E}}$ is the polarization of the photon rotated by the $\rho^{\prime}$ c.m.s. production angle about the production normal; the other quantities are defined in Footnote 8 . We find that $J^{P}=1^{-}$and phase space is required to fit the data; $2^{-}$is rejected by the fit. ${ }^{9}$ The curves on Fig. 1d and Fig. 2a show that the matrix element (3) describes the data well.

Cross Section for s-Channel Helicity Conserving $4 \pi 1^{-}$States

For the decay matrix element (3) we have calculated, using Monte Carlo events, the efficiency of the analyzer $\vec{Q}$; the efficiency is $70 \%$ at $\mathrm{M}_{4 \pi} \sim 1.5 \mathrm{GeV}$, and falls to $40 \%$ at $2.6 \mathrm{GeV}$. We use this efficiency to correct the values of $I$ (Fig. 2b) obtained experimentally and display the result in Fig. 1a. The corrected II peaks at $\sim 1.5 \mathrm{GeV}$ and has a FWHM of $\sim 0.60 \mathrm{GeV}$; it accounts for 
practically all events with $\mathrm{M}_{4 \pi}$ less than $1.5 \mathrm{GeV}$. The production cross section calculated from the corrected II is $1.3 \pm 0.4 \mu \mathrm{b}$. We note that the corresponding cross section at $4.7 \mathrm{GeV}$ is $0.8 \pm 0.4 \mu \mathrm{b}$.

Next we estimate the upper limit for decay of the $\rho^{\prime}$ into $\pi^{+} \pi^{-}$and $\mathrm{K}^{+} \mathrm{K}^{-}$ from $\Sigma \operatorname{Re} \mathrm{Y}_{2}^{2}(\theta, \psi)$ for $\gamma \mathrm{p} \rightarrow \pi^{+} \pi^{-} \mathrm{p}$, and $\mathrm{K}^{+} \mathrm{K}^{-} \mathrm{p}$. We use the mass interval $1.15-1.75 \mathrm{GeV}$ and the t-interval $0.02-1.0 \mathrm{GeV}^{2}$. The resulting upper limits (2 standard deviations) are $\rho^{\prime} \rightarrow \pi^{+} \pi^{-} / \rho^{\prime} \rightarrow \rho^{\circ} \pi^{+} \pi^{-}=0.2$, and $\rho^{\prime} \rightarrow \mathrm{K}^{+} \mathrm{K}^{-} / \rho^{\prime} \rightarrow \rho^{\mathrm{o}} \pi^{+} \pi^{-}=0.04$.

Maximum Likelihood Fits

We have attempted to obtain a better estimate of the $\rho^{\prime}$ cross section by making an overall maximum-likelihood fit to reaction (1) including, besides the $\rho^{\prime}$, the channels $\Delta^{++} 3 \pi, \Delta^{++} \rho^{o} \pi^{-}, \Delta^{++} A_{2}^{-}, \rho^{o} \pi^{+} \pi^{-} p$, and phase space. The $\rho^{\prime}$ is parameterized by a Breit-Wigner of the form $\exp (\mathrm{At} / 2) /\left(\mathrm{M}_{\rho^{\prime}}, \mathrm{M}_{4 \pi}-\frac{i}{2} \gamma_{\rho^{\prime}} \cdot \frac{\mathrm{K}\left(\mathrm{M}_{4 \pi}\right)}{\mathrm{K}\left(\mathrm{M}_{\rho^{\prime}}\right)}\right)$ which multiplies the RHS of Eq. (3). K describes the energy dependence of the width, ${ }^{10}$ and $\mathrm{A}$ is the slope of the t-distribution. The amplitudes of the other channels are described in Ref. 3.

We obtain a good description of the channel, ${ }^{11}$ as shown by the curves on the mass and decay distributions in Figs. 1 and 2. For the $\rho^{\prime}$ mass and width $\left(\gamma_{\rho^{\prime}}\right)$ we obtain $1.43 \pm 0.05 \mathrm{GeV}$ and $0.65 \pm 0.1 \mathrm{GeV}$, respectively. We note however that these quantities are sensitive to the choice of the function $\mathrm{K} .{ }^{3} \mathrm{We}$ find a cross section for $\rho^{\prime} \rightarrow \rho^{0} \pi^{+} \pi^{-}$of $1.6 \pm 0.4 \mu \mathrm{b}$, which is insensitive to $\mathrm{K}$. The slope of the $\rho^{\prime} \mathrm{t}$-distribution is $5.6 \pm 0.3 \mathrm{GeV}^{-2}$. Conclusion

We have confirmed the existence of a $4 \pi$ enhancement at a mass $\sim 1.5 \mathrm{GeV}$ and have shown that it is an $\mathrm{I}^{\mathrm{G}}=1^{+}, \mathrm{J}^{\mathrm{P}}=1^{-}$state which decays primarily into $\rho^{\mathrm{o}} \pi^{+} \pi^{-}$. It is photoproduced via natural parity exchange with a cross section of 
$\sigma\left(\gamma \mathrm{p} \rightarrow \mathrm{p} \rho^{\dagger} \rightarrow \mathrm{p}^{\mathrm{o}} \pi^{+} \pi^{-}\right)=1.6 \pm 0.4 \mu \mathrm{b}$. The production mechanism is predominantly s-channel helicity-conserving.

\section{Acknowledgements}

We wish to thank the SLAC Operations Crew, and R. Watt and the $82^{\prime \prime}$ Bubble Chamber Operations Group for their assistance in performing this experiment. We thank R. Gearhart, J. J. Murray, and C. K. Sinclair for their help during the early stages of the experiment. We acknowledge the diligence of our scanners and data reduction group, in particular D. Blohm, K. Eymann, W. Hendricks and A. Wang. 


\section{REFERENCES}

1. M. Davier et al., contribution to the Symposium on Electron and Photon Interactions at High Energies, Cornell, 1971; SLAC-PUB-666 (1969) (unpublished).

2. G. Bacci et al., Phys. Letters 38B, 551 (1972); G. Barbarino et al., Lettere al Nuovo Cimento $\underline{3}, 689$ (1972).

3. G. Smadja et al., Lawrence Berkeley Laboratory Report LBL 991 and Proceedings of the 1972 Philadelphia Conference on Meson Spectroscopy (Am.Inst. of Phys. 1972).

4. J. Ballam et al., Phys. Rev. D5, 545 (1972) and to be submitted for publication.

5. C. K. Sinclair et al., IEEE Trans. on Nucl. Sci. 16, No. 3, 1065 (1969). W. J. Podolsky, LRL Berkeley Report, UCRL-20128 (1971), (Ph.D. thesis, unpublished).

6. H. H. Bingham et al., "Total and partial $\gamma p$ cross sections at $9.3 \mathrm{GeV}, "$ to be submitted to Phys. Rev.

7. We analyze the vector meson (VM) decay in the helicity frame, where the $z$ axis is the direction of the VM in the overall ( $(\gamma p)$ CMS. The y axis is the normal to the production plane, defined by the cross product $\hat{\mathrm{k}} \times \hat{\mathrm{v}}$ of the directions of the photon and the vector meson. The $\mathrm{x}$ axis is given by $\hat{x}=\hat{y} \times \hat{z}$. The angle $\Phi$ between the polarization of the photon, $\hat{\epsilon}$, and the production plane in the total c.m. system is defined by $\cos \Phi=\hat{\epsilon} \cdot(\hat{y} \times \hat{k})$, $\sin \Phi=\hat{\mathrm{y}} \cdot \hat{\epsilon}$. The decay angles $\theta, \phi$ are the polar and azimuthal angles of the appropriate analyzer $\hat{n}$ : for two body decays we use the direction of one of the decay mesons in the vector meson CMS; for $3 \pi$ decay the decay plane normal is used. For $4 \pi$ decay we use the vector sum of the two $\pi^{+}$ 
momenta in the $4 \pi$ CMS (see text for discussion): $\cos \theta=\hat{n} \cdot \hat{z}$, $\cos \phi=\hat{y} \cdot(\hat{z} \times \hat{n}) /|\hat{z} \times \hat{n}|, \sin \phi=-\hat{x} \cdot(\hat{z} \times \hat{n}) /|\hat{z} \times \hat{n}|$, and $\psi=\phi-\Phi$. In the forward direction $\psi$ is the angle between the photon polarization and the meson decay plane.

8. For a state of spin-parity $\mathrm{J}^{\mathrm{P}}$ decaying into $\rho^{\sigma}$ with relative angular momentum $\ell$, we use amplitudes of the form $T=\sum_{i=1}^{4}\left(e^{-i \Phi} A_{i}(J, 1)-P(-1)^{J} e^{i \Phi} A_{i}(J,-1)\right)$ with

$$
A_{i}(J, M)=p^{\ell} q_{i} \sum_{m m^{\prime}} c\left(J M ; l m 1 m^{\prime}\right) Y_{\ell}^{m}(\theta, \phi) Y_{1}^{m^{\prime}}\left(\theta^{\prime}, \phi^{\prime}\right) \frac{\sin \delta_{i}^{p} e^{i \delta_{i}^{p}}}{q_{i}^{3}} \frac{\sin \delta^{s} e^{i \delta}}{q}
$$

The summation over i symmetrizes the amplitude over all $\pi^{+} \pi^{-}$combinations.

$\Phi$ is defined in Footnote $7 . p, \theta, \phi$ are respectively the momentum, polar angle, and azimuth of the $\rho$ in the $\rho^{\prime} \mathrm{CMS} ; \mathrm{q}_{i}, \theta^{\prime}, \phi^{\prime}$ are respectively the momentum, polar angle, and azimuth of the $\pi^{+}$resulting from the decay of the ith $\rho$ in the $\rho$ rest frame; $q$ is the momentum of the $\pi^{+}$in the rest frame of the $\sigma$ opposite to the ith $\rho . \mathrm{Y}_{1}^{\mathrm{m}^{\prime}}\left(\theta^{\prime}, \phi^{\prime}\right), \mathrm{Y}_{\ell}^{\mathrm{m}}(\theta, \phi)$ are spherical harmonics describing the decay $\rho \rightarrow \pi^{+} \pi^{-}$and $\rho^{\prime} \rightarrow \rho^{\sigma}$ respectively. The $\mathrm{I}=1 \mathrm{p}$-wave and $\mathrm{I}=0 \mathrm{~s}$-wave dipion phase shifts are given by $\delta^{\mathrm{p}}$ and $\delta^{\mathrm{s}}$ respectively. We use the phase shifts of S. D. Protopopescu et al., LBL 787 (1972) and Proceedings of Philadelphia Conference on Meson. Spectroscopy (1972), to be published.

9. Fitting $J^{P}=1^{-}$and phase space alone gives a $P\left(\chi^{2}\right)$ of $80 \%$ in the $\psi$ distribution, whereas a fit of $2^{-}$and phase space alone gives a $P\left(\chi^{2}\right)<1 \%$.

10. We use $\mathrm{K}=\mathrm{G}\left(\mathrm{M}_{4 \pi}\right) /\left(1+\mathrm{G}\left(\mathrm{M}_{4 \pi}\right) / \mathrm{G}\left(\mathrm{M}_{\rho},\right)\right)$ where $G\left(M_{4 \pi}\right)=\frac{1}{M_{4 \pi}} \int \mid$ Breit-Wigner $\left.\right|_{\rho} ^{2} \prod_{i=1}^{4} \frac{d^{3} p_{i}}{2 E_{i}} \delta_{4}\left(p-\sum_{i=1}^{4} p_{i}\right)$ 
This approaches a constant at large $4 \pi$ masses in contrast to the parameterization used in Ref. 3 .

11. The fitted percentages of the channels $\mathrm{p} \rho^{\prime}, \Delta^{++} 3 \pi, \Delta^{++} \rho^{\mathrm{o}} \pi^{-}, \Delta^{++} \mathrm{A}_{2}^{-}$, $\rho^{0} \pi^{+} \pi^{-} \mathrm{p}$, and phase space are $40 \pm 8,16 \pm 4,25 \pm 6,7 \pm 2,3 \pm 3,10 \pm 4$, respectively.

\section{FIGURE CAPTIONS}

1. Reaction $\gamma p \rightarrow 2 \pi^{+} 2 \pi^{-} p$ (a) Four pion mass spectrum. The shaded histogram has events with $\Delta^{++}$removed $\left(\mathrm{M}_{\mathrm{p}_{\pi}+}>1.32 \mathrm{GeV}\right)$. The points are $\Pi$ (see Eq. (2)) corrected for efficiency. The curve is from a maximumlikelihood fit to the channel. (b) $\pi^{+} p$ mass distribution. The shaded events are for $\mathrm{M}_{4 \pi}<1.7 \mathrm{GeV}$. The curve is from the maximum-likelihood fit. (c) $\pi^{+} \pi^{-}$mass distribution $\left(\mathrm{M}_{4 \pi}<1.7 \mathrm{GeV}\right)$ for $\pi^{+} \pi^{-}$pairs opposite a $\rho^{\circ}$. The dotted (solid) curve shows the distribution expected for $\rho^{0} \rho^{o}\left(\rho^{o} \pi^{+} \pi^{-}+\right.$phase space). (d) $\pi^{+} \pi^{-}$mass distribution. The shaded events are for $\mathrm{M}_{4 \pi}<1.7 \mathrm{GeV}$. The curve is from the maximum-likelihood fit.

2. (a) Distribution of the angles $\theta$ and $\psi$ for $\mathrm{M}_{4 \pi}<1.7 \mathrm{GeV}$. The curve is from the maximum-likelihood fit. (b) II uncorrected for analyzer efficiency. 


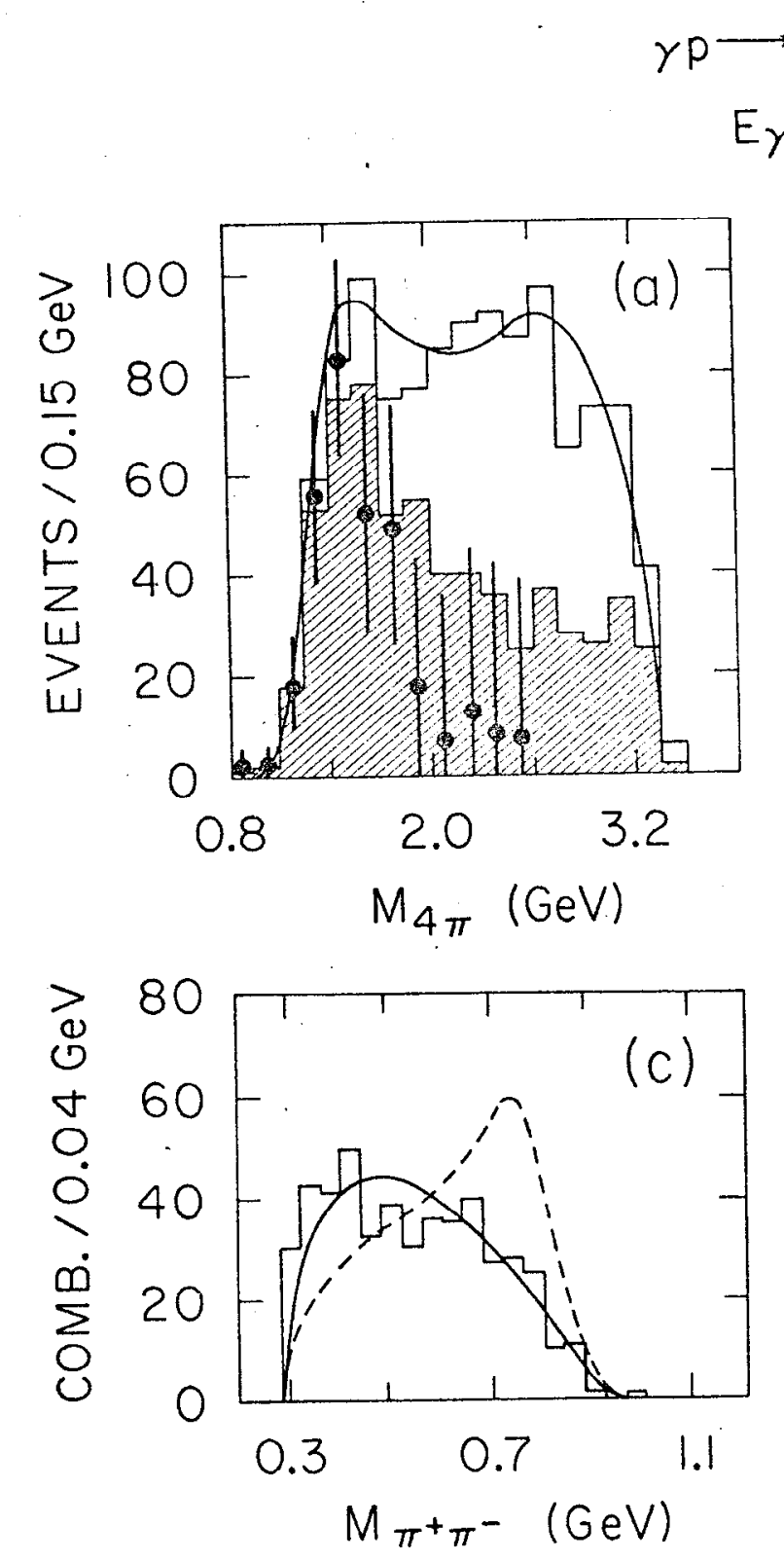

$E_{\gamma}=9.3 \mathrm{GeV}$
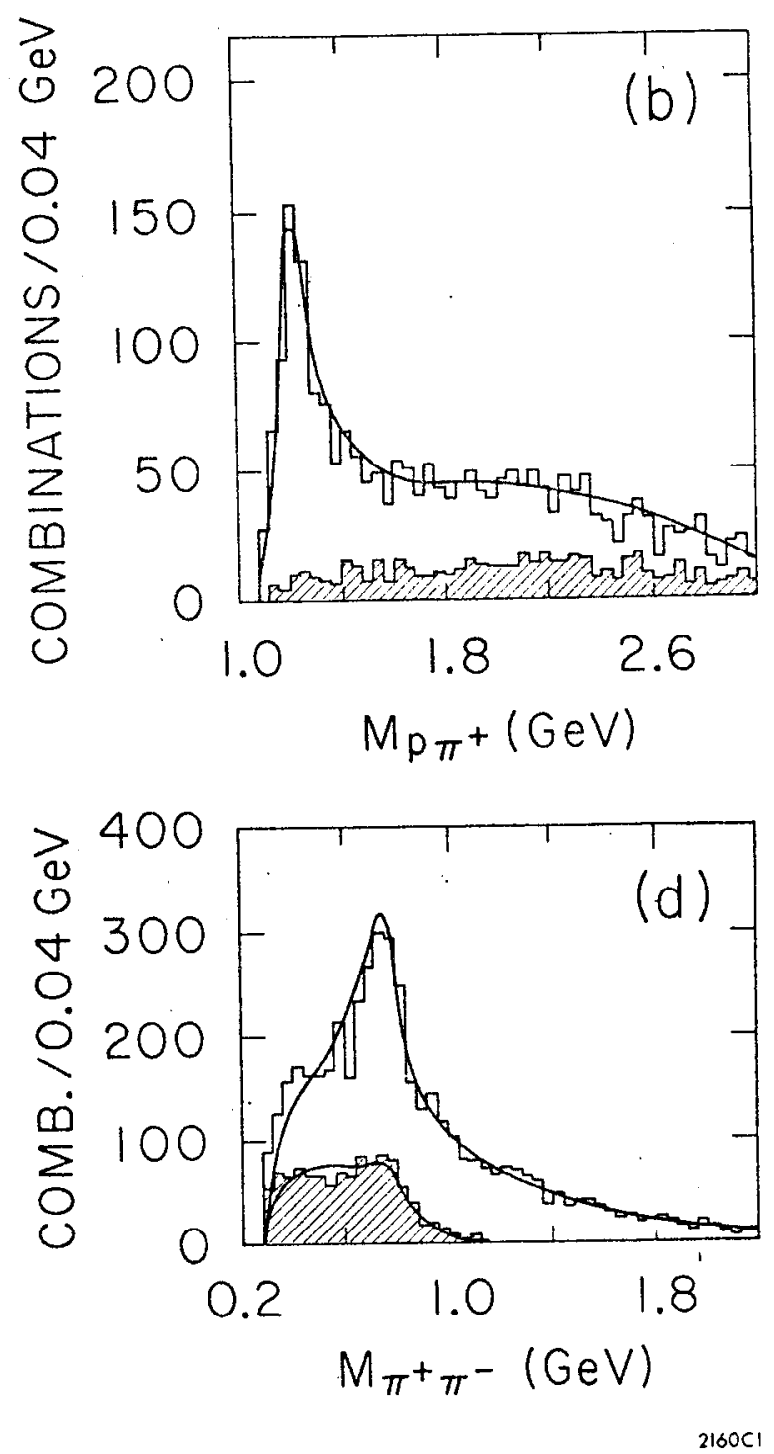

Fig. 1 

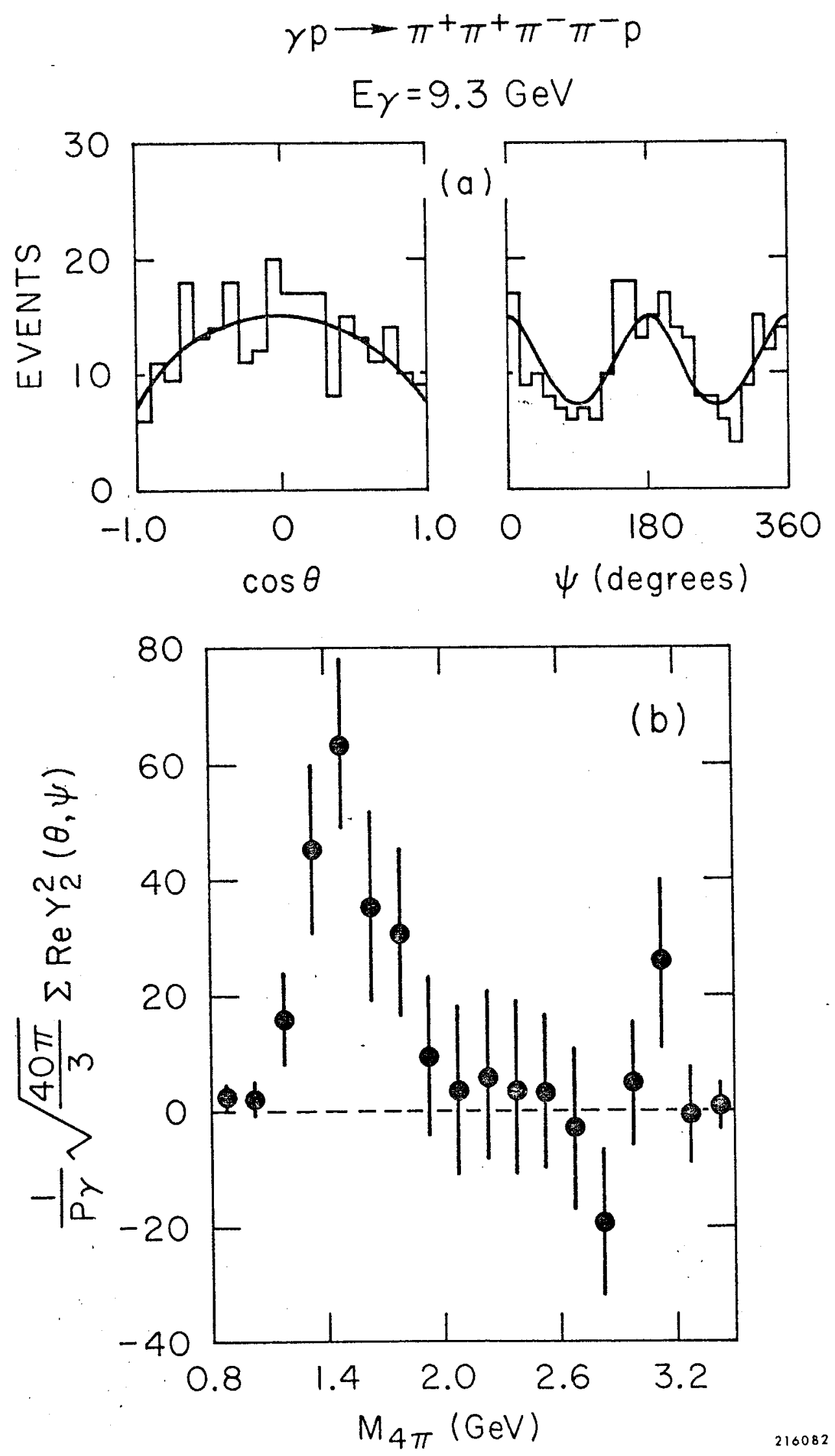

216082

Fig. 2 